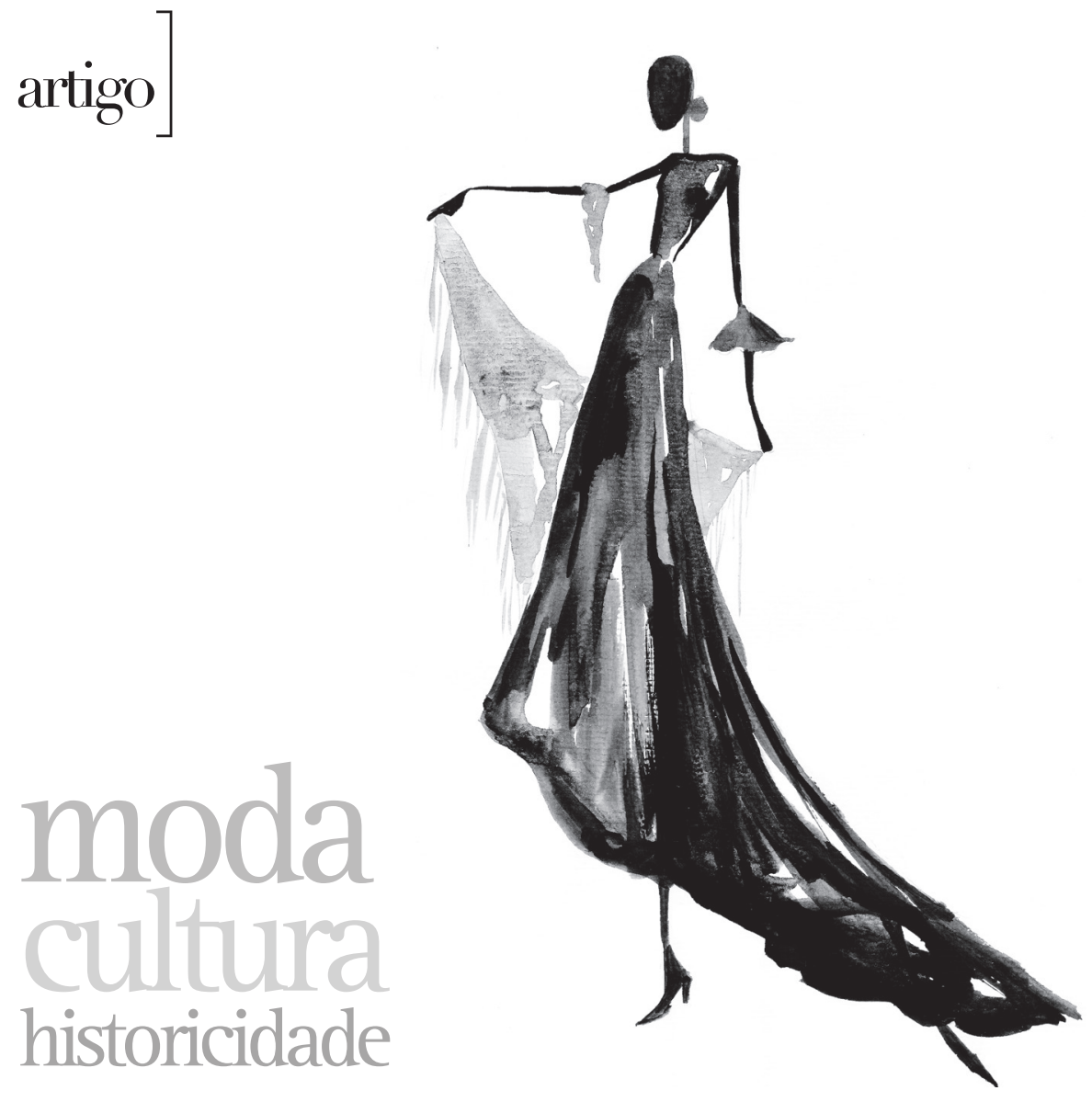

0 Grupo de Trabalho Moda, Cultura e Historicidade discute questões relativas ao estudo da história da moda e da cultura das aparências. As pesquisas apresentadas e debatidas neste grupo tratam de temas como: interfaces entre arte, moda e design; relações entre moda, aparência, gênero e identidade numa perspectiva histórica; cultura, consumo e indústria da moda; transformações e/ou permanências das formas vestimentares; moda e cultura visual.

0 artigo de Maria Cristina Volpi, As roupas pelo avesso: cultura material e história social do vestuário, relata uma experiência de trabalho, muito bem realizada, de organização de acervo de indumentária. Apresenta um projeto de formação de acervo para estudo, demonstrando a metodologia e a base de referência bibliográfica empregadas em cada etapa de desenvolvimento da pesquisa. Além disso, oferece exemplos e discute indumentária como linguagem, constituindo um estímulo aos profissionais de diferentes instituições para que empreendam iniciativas congêneres. Destaca-se ainda pelas contribuições aos campos da teoria da moda e metodologia de pesquisa.

Rita Andrade e Maria do Carmo Rainho 
[ MARIA CRISTINA VOLPI ]

Professora associada da Escola de Belas Artes e do Programa de Pós-Graduação em Artes Visuais da Universidade Federal do Rio de Janeiro onde desenvolve pesquisas no campo da história da indumentária e da moda no Brasil. Publicou, entre outros, Cosmopolitismo e vestuário masculino na Belle Époque no Rio de Janeiro na revista Arte \& Ensaios (n. 25, maio 2013).

E-mail: mcvolpi@ufrj.br

\title{
As roupas pelo avesso: cultura material e história social do vestuário
}

\author{
The clothes inside out: material culture \\ and social history of fashion
}

[resumo] Este ensaio discute o estudo da indumentária em acervos de objetos, tendo como exemplo a organização do acervo de vestuário e acessórios do Centro de Referência Têxtil/Nestuário, projeto que coordeno desde 2005 que visa ao desenvolvimento de pesquisas sobre as formas vestimentares na Escola de Belas Artes da Universidade Federal do Rio de Janeiro.

\section{palavras-chave $]$}

materioteca; cultura material; formas vestimentares.

[abstract] This essay discusses the study of clothing in collections of objects, taking as an example the organization of the clothing and accessory collection of the Centro de Referência Têxtil/Vestuário (Textile/Clothing Reference Center), a project I have been coordinating since 2005, aimed at developing research on ways of dressing at the Escola de Belas Artes (School of Fine Arts) of the Universidade Federal do Rio de Janeiro.

[keywords] resource center; material culture; ways of dressing. 


\section{Para uma história da indumentária}

Fato antropológico quase universal, a indumentária' situa-se ao lado da linguagem e da arte como prática significante e, como objeto, faz parte do conjunto de instrumentos por meio dos quais o homem interfere no ambiente natural, domínio da cultura material. A produção de sentido se dá através de sua estética, expressa pelas matérias, cores e formas empregadas na construção da indumentária e, ao mesmo tempo, pela ligação intelectual e afetiva que se estabelece entre o traje e seu usuário. Seus aspectos plásticos não se reduzem a termos puramente estáticos, uma vez que o corpo está em movimento.

Não só a forma e o movimento do corpo servem como referencial para a elaboração dos trajes, mas também a moda: a partir do Renascimento, a moda é um fenômeno social característico do Ocidente que vem regular as formas vestimentares. Tendo como metáfora perfeita o vestuário, é frequentemente confundida com ele. Nas sociedades modernas, a mudança contínua nas formas dos trajes e acessórios é o elo entre o individual e o coletivo (BURGUELIN, 1995).

A moda faz parte do universo de signos urbanos desde sua origem. Sua variação constante e a tipologia prescrita pela educação formal e pelos agentes legitimadores do padrão dominante associam códigos de civilidade, práticas sociais e os lugares da cidade onde são usados esses trajes. Desse modo, se articula a aparência vestida e o espaço urbano transformado em cenário, contribuindo para estreitar a relação entre a cidade e a moda.

0 estudo da indumentária, sistema vestimentar formal e normativo de uma sociedade, formado por elementos que compõem a aparência vestida (ROCHE, 2000), fundamenta-se na investigação de diversos tipos de documentos que podem ser sintetizados nos três vestuários de Barthes (1981): o vestuário escrito formado pela descrição do traje cuja estrutura é verbal, 0 vestuário imagem que é desenhado ou fotografado cuja estrutura é plástica e o vestuário real cujas marcas das ações de fabrico formam uma estrutura tecnológica. Ao repertório dos textos encontrados em arquivo (de notários, comerciantes, fabricantes e familias) e das fontes iconográficas (pinturas, estampas, gravuras e fotografias) somam-se os acervos de vestuário e acessórios. Nesse contexto, o traje e os acessórios são documentos fundamentais para o estudo da história do vestuário e da moda, "[...] suporte material, fisico, imediatamente concreto, da produção e reprodução da vida social" (MENESES, 1983, p. 112).

Estudos mais recentes sobre a indumentária fundamentam-se sobre a fusão entre o estudo do objeto e as abordagens teóricas (TAYLOR, 2004). Além do entendimento de seus contextos históricos mais amplos, 
a partir dos objetos pode-se compreender como são feitos, para que são usados, como se dá a circulação dos materiais e modelos e quais são os seus valores expressivos em termos culturais e estéticos. Essas novas abordagens contribuíram para que o trabalho do historiador se enriquecesse.

A historiografia da indumentária no Brasil é bastante recente, e estudos com foco em acervos de vestuário são mais raros ${ }^{2}$. No Rio de Janeiro praticamente todos os museus da cidade têm alguma peça de vestuário em seu acervo, embora a maioria não ultrapasse o início do século XIX. Tanto a cidade quanto o Estado do Rio de Janeiro possuem importantes acervos públicos ${ }^{3}$. Num levantamento inicial realizado na Universidade Federal do Rio de Janeiro (UFRJ) destacam-se as coleções de indumentária indígena e têxteis arqueológicos no Museu Nacional na Quinta da Boa Vista, além de um pequeno acervo de objetos pessoais incluindo leques, relógios, joias e trajes eclesiásticos que fazem parte da Coleção Ferreira das Neves do Museu Dom João VI da Escola de Belas Artes.

0 projeto do Centro de Referência Têxtil/Nestuário (CRTV) vem sendo desenvolvido desde 2005 com o objetivo de oferecer subsídios para o ensino e a pesquisa sobre a história da indumentária no Brasil. Está vinculado ao Núcleo Interdisciplinar de Estudo da Imagem e do Objeto/NIO do Programa de Pós-Graduação em Artes Visuais/PPGAV e envolve a participação de estudantes de graduação e pós-graduação na organização de materiotecas e banco de imagens.

\section{Estratégias para formar o acervo}

0 acervo, que começou a ser formado a partir de doações de peças de trajes civis dos séculos XIX e XX, possui atualmente 108 itens entre vestidos, casacos, ternos, fraques, coletes, camisolas, anáguas, combinações, saias, sapatos, bolsas, cintos, chapéus, luvas, além de caixas de chapéus. Em razão da política de aquisição dos parâmetros estabelecidos para sua constituição e do espaço disponível, cada traje recebido é examinado pela equipe, sendo aproveitados aqueles que têm mais relevância e por seu estado de conservação. A partir de pesquisas desenvolvidas pelos estudantes foram elaboradas as fichas ${ }^{4}$ de doação, de coleta de dados e de catalogação que reúnem informações necessárias para subsidiar as pesquisas.

As peças são guardadas no mobiliário existente na sala 709 da Escola de Belas Artes, formado por armários e mapotecas, adaptados para esse fim. Para 
o acondicionamento das peças levou-se em conta a melhor forma de armazenagem, plana ou vertical, dependendo do formato e material de que são feitas.

$\mathrm{Na}$ armazenagem plana foram utilizadas as gavetas, forradas com polionda, a fim de evitar que a madeira entrasse em contato direto com os trajes. Roupas feitas de renda ou cambraia, os itens menores, como as luvas, roupas de baixo e trajes infantis, foram acondicionados nas gavetas.

Peças de vestuário, como casacos, paletós e vestidos, foram armazenadas verticalmente em cabides de plástico acolchoados, forrados com algodão cru, e cobertas com capas de algodão cru, em largura padrão e comprimento de acordo com o formato da peça a ser guardada. Essa capa tem uma janela na lateral, forrada com poliéster transparente, para que se possa ver uma parte do traje, e um bolso para ser incluída uma fotografia da peça, permitindo sua identificação sem 0 manuseio direto.

As rotinas de trabalho do acervo de trajes e acessórios foram estabelecidas e consolidadas, visando garantir a continuidade dos trabalhos. A capacitação constante dos estudantes envolvidos no projeto se dá por intermédio de parcerias com setores na UFRJ e de outras instituições. São feitas visitas técnicas a museus no Rio de Janeiro e em São Paulo, e incentivadas participações dos estudantes em seminários e cursos que possam contribuir para as ações necessárias de coleta, acondicionamento, guarda, indexação e recuperação de dados.

\section{Aporte teórico-metodológico empregado}

Para a indexação das peças de vestuário no acervo, além do uso do Thesaurus para acervos museológicos (FERREZ e BIANCHINI, 1987), foi necessário definir um sistema descritivo adequado, a partir das propostas de Balfet (1984) e Duflos-Priot (1984).

Os parâmetros utilizados para elaborar um léxico que sirva para a classificação e recuperação por acesso ao banco de dados on-line dessas diferentes peças museológicas levam em conta as caracteristicas materiais e simbólicas das roupas. As zonas de apoio da peça no corpo foram tomadas como elementos de definição da roupa, a partir das quais foram listadas as características formais do vestuário, ou seja, as partes do traje. Além disso, são descritas as matérias e as técnicas empregadas para 0 fabrico. No sistema descritivo utilizado, as classificações não deverão ser rígidas e poderão receber novos termos.

As peças foram fotografadas e após o armazenamento o número de inventário (siglado) é fixado em cada uma. As fichas estão em fase de construção, incluindo a realização de um desenho técnico e o levantamento de referências bibliográficas. 
A cada doação são colhidos dados em uma ficha de cadastro do doador e outra ficha do usuário. Cada peça possui uma ficha com o máximo de informações colhidas com o doador sobre o perfil sociocultural do usuário, com dados sobre gênero, idade, profissão e em que circunstâncias a roupa foi usada. Uma vez que os trajes classificam-se também por sua relação com as ocasiões em que são usados, cada uma das vivências associadas à indumentária contribui para situar o indivíduo e sua indumentária e a sociedade a que pertence.

Sempre que possível são vinculadas às peças doadas fotografias das pessoas que as usaram, como a combinação de cambraia bordada que pertenceu a uma das irmãs Lahmeyer retratadas na Carte de Cabinet datada dos anos 1880 .

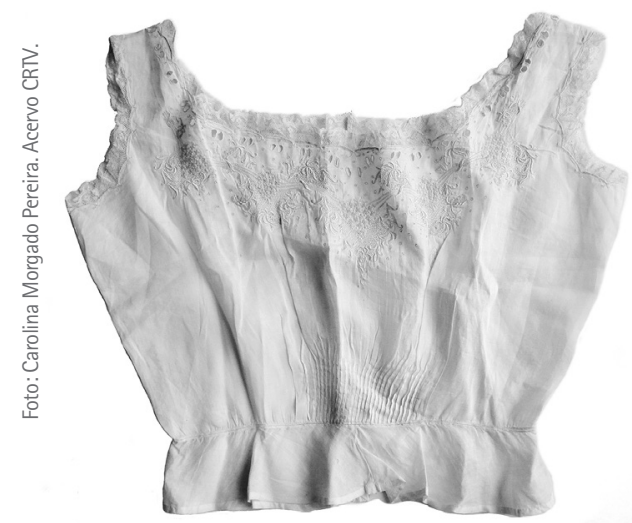

0 retrato de Ida Catarina, Laura Maria e Cecilia Salomé Lahmeyer foi realizado por uma tradicional empresa do ramo situada no centro do Rio de Janeiro. Para estimar a data, foram considerados tanto os dados obtidos sobre as pessoas retratadas quanto a forma geral da vestimenta. Na frente da fotografia está impresso na margem inferior: "Tavares Sobrinho Succ [sucessor] de Carneiro \& Gaspar"; no verso: "Tavares Sobrinho sucessor de Carneiro \& Gaspar 54 Rua de Gonçalves Dias 54 Rio de Janeiro D. Hutinet Paris". Desse mesmo lado, escrito a caneta por uma das filhas de Cecilia, lê-se: "Da esquerda para direita - tia Ida - Dindinha - Mamãe ou Ida Catarina, Laura Maria, Cecilia Salomé". Se as anotações informam qual o nome de cada uma, outros dados foram recolhidos com o doador, como o fato de as três moças serem irmãs. Por outro lado, a análise da imagem, das roupas usadas, das joias e dos outros complementos, a própria fotografia, seu formato e a agência que a produziu denotam o capital social. 

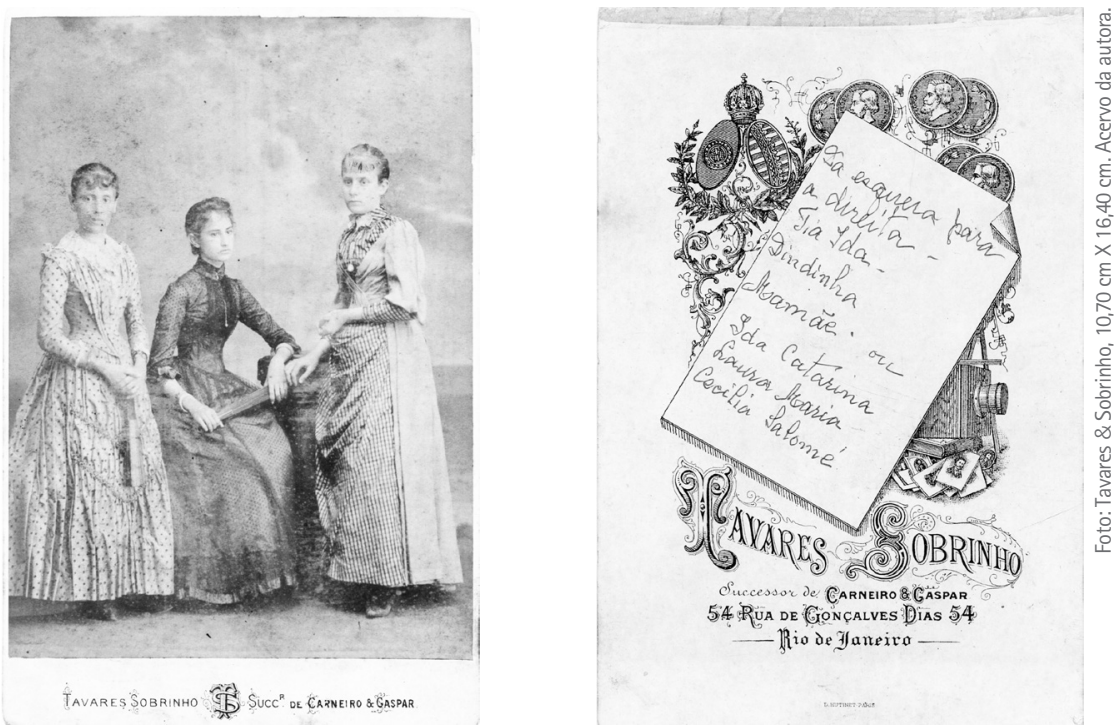

Figura 2: Carte de Cabinet, frente e verso, c. $1880^{5}$. Exemplo de fotografia de usuário.

Algumas vezes é possível encontrar o usuário envergando a peça doada. É o caso do vestido da década de 1960 feito de seda estampada em tons de marrom e laranja, sem mangas, com decote redondo na frente e atrás, com saia rodada, abaixo do joeIho, usado com bolero de mangas com gola xale do mesmo tecido do vestido e forrado de seda laranja.

Doado por Marta Isabel Gomes Gonçalves de Mello, o vestido de coquetel pertenceu à sua irmã, Claudia Ribeiro Gomes, retratada por ocasião de um desfile de moda realizado no Rio de Janeiro.

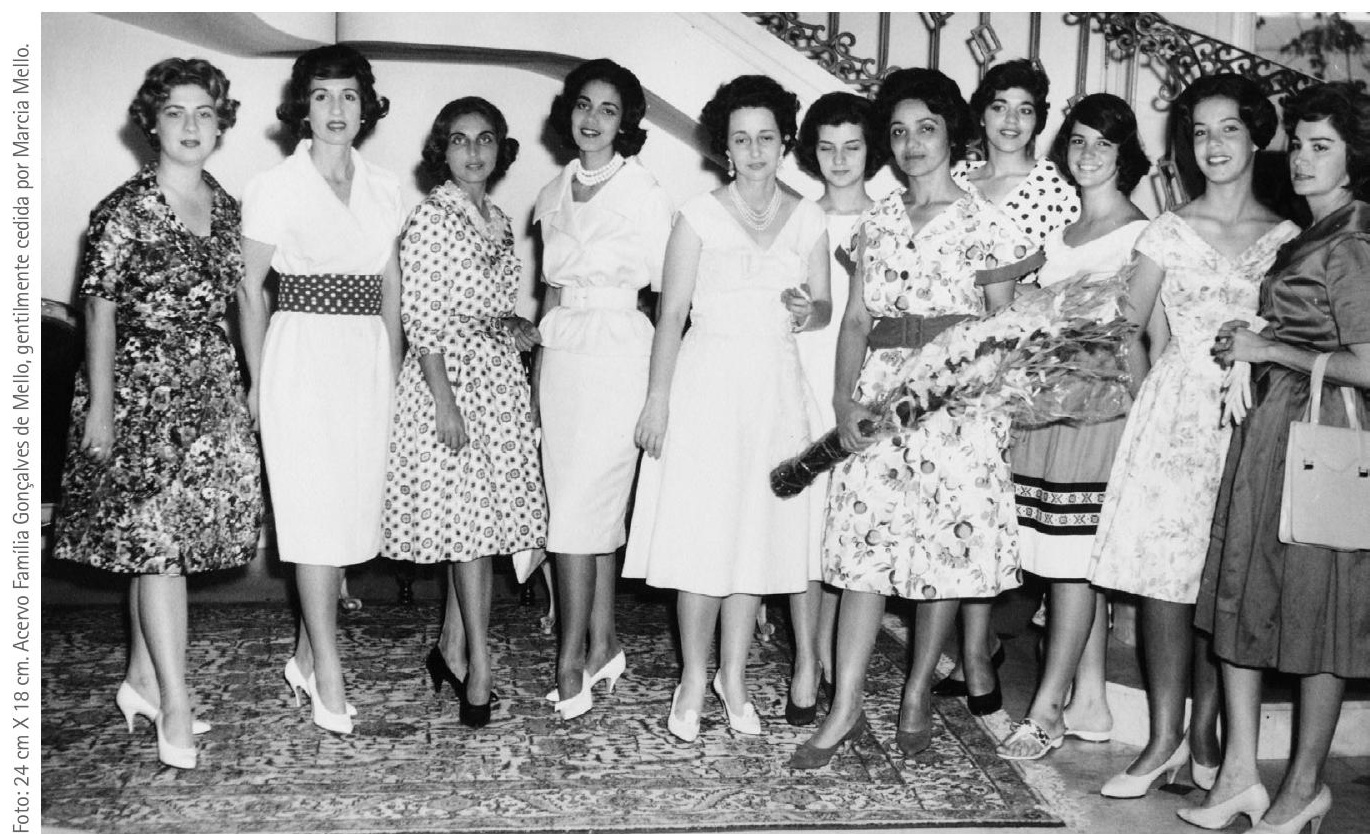

Figura 3: Desfile de moda na década de 1960. Claudia Ribeiro Gomes é a primeira à esquerda.

Nesse caso a composição formada pelo vestido, o sapato e o penteado, além do cenário no qual o vestido foi usado, são dados suplementares que revelam práticas nas quais o objeto se insere.

Algumas peças são doadas em caixas originais, a exemplo de dois chapéus masculinos da década de 1930, uma delas da Casa Garcia, situada na Avenida Rio Branco, $n^{\circ} 93$ a 97, Rio de Janeiro, e outra, dos fabricantes de feltro Vicente Cury e Cia., de Campinas (SP), ambas em estilo art déco. Outras contêm pequenas anotações, como a caixa na qual foi guardada a roupa de batizado masculina dos anos 1950, com a frase "feito pela mamãe" escrita a lápis numa das faces. 
Estão inscritas na materialidade das peças ações de fabrico e uso, registros de memória que revelam corpos e afetos. As propriedades do traje, evidenciadas pelo tipo de tecido, modelagem, técnicas de costura e bordado empregados, se completam com essas pequenas informações que chegam junto com as embalagens ou, ainda, com as particularidades do usuário fotografado. Tais dados não só revelam o investimento afetivo, mas também contribuem para determinar as características da camada social associada à prática vestimentar.

Tanto as peças de vestuário quanto as embalagens onde foram guardadas, fotografias dos usuários, anotações e relatos colhidos com as pessoas que doaram as peças fazem parte do conjunto material e imaterial a ser analisado, a partir da fundamentação teórica dos estudos de cultura material aplicados à história social, segundo os quais os objetos encarnam padrões de crenças e comportamentos (PROWN, 1982). A metodologia empregada para análise é formada por três etapas: a) descrição (análise substancial, conteúdo e análise formal); b) dedução (experiência sensorial, experiência intelectual e resposta emocional); e c) especulação (formulação de hipóteses, programa de pesquisa) associada à investigação das evidências externas (análises quantitativa, estilística e iconológica).

Os indicadores sociais são analisados a partir das propostas teóricas de Pierre Bourdieu (1989, p. 134) para quem:

As propriedades actuantes, tidas em consideração como princípios do espaço social, são as diferentes espécies de poder ou de capital que ocorrem nos diferentes campos. 0 capital - que pode existir no estado objectivado, em forma de propriedades materiais, ou, no caso do capital cultural, no estado incorporado, e que pode ser juridicamente garantido - representa um poder sobre um campo (num dado momento), e mais precisamente, sobre o produto acumulado do trabalho passado (em particular sobre o conjunto dos instrumentos de produção), logo sobre os mecanismos que contribuem para assegurar a produção de

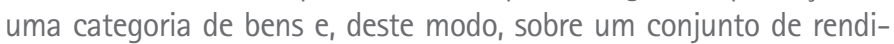
mentos e ganhos [...].

As histórias de vida, o patrimônio econômico, cultural e social contribuem para o estudo histórico da aparência, para a apreensão do capital social do usuário e da sua visão de mundo.

\section{Três vezes indumentária}

Colecionar amostras ou fazer levantamentos pode ser uma tarefa sem fim, por isso mesmo foram estabelecidos critérios quanto ao escopo e abrangência da coleção a ser formada. A coleta de dados e a organização do acervo foram orientadas de modo a articular as três categorias documentais propostas por Barthes (1981), contribuindo para que o estudo das peças se dê da forma mais acurada possivel. Desse modo, a organização da materioteca traz um benefício adicional por favorecer a vivência e integrar o aprendizado a partir dos objetos e do entendimento de seus contextos históricos mais amplos.

As questões que emergem da experiência com a "coisa real" são

[...] um esforço constante para fazer falar as coisas mudas, para fazê-las falar o que elas não falam sobre si mesmas, sobre os homens e as sociedades que as produziram, utilizaram, transformaram, aperfeiçoaram a seu modo. ${ }^{6}$ (FEBVRE, 1949, p. 236, tradução nossa)

Ao nos debruçar sobre esse pequeno acervo, articulando relatos, histórias de vida, leitura de imagens e de objetos, procuramos contribuir singelamente para uma escrita da história da indumentária no Brasil. 


\section{NOTAS}

${ }^{[1]} 0$ termo aqui é empregado no sentido de arte do vestuário, história do vestuário, uso do vestuário em relação às épocas e aos povos (FERREIRA, 2010).

[2] 0 Núcleo Interdisciplinar de Estudos de Moda (NIDEM) foi criado em 1997 pela socióloga Solange Wajnman, vinculado à Universidade Paulista, campus Paulista em São Paulo (SP), tendo como finalidade reunir e divulgar as pesquisas acadêmicas sobre moda, articulando os campos de história, antropologia, sociologia e comunicação. 0 Colóquio de Moda, realizado anualmente desde 2005, reúne um grupo de professores e pesquisadores vinculados a instituições de ensino e pesquisa brasileiras e constituise num importante fórum para divulgação e discussão de pesquisas multidisciplinares sobre vestuário e moda. Nesse âmbito, o Grupo de Trabalho Moda, Cultura e Historicidade, do qual faço parte, trata da produção historiográfica sobre moda e cultura das aparências. Como resultado desses debates, foi publicado História e cultura de moda (BONADIO e MATTOS, 2011). Em 2011, durante o I Encontro Nacional de Pesquisa em Moda e VI FAV Fashion na Faculdade de Artes Visuais da Universidade Federal de Goiás, foram apresentados trabalhos sobre a pesquisa em acervos têxteis, no eixo temático "Têxteis, materialidade, visualidade, sentidos e patrimonialização", sob a coordenação de Rita Andrade (FAV/UFG) - uma das primeiras pesquisadoras a empregar o traje como corpus principal em suas pesquisas - e Manuelina Duarte (FCS/UFG), privilegiando o estudo de coleções de museus como fontes primárias.

${ }^{[3]}$ Segundo o Guia de Museus Brasileiros (2000), na cidade do Rio de Janeiro os principais acervos públicos com coleções de trajes e acessórios são: Museu Histórico Nacional, Museu da República, Museu Carmen Miranda, Museu Casa de Benjamin Constant, Fundação Casa de Rui Barbosa, Museu da Academia Nacional de Medicina, Museu da Imperial Irmandade de Nossa Senhora da Glória do Outeiro, Museu Sacro, Museu da Polícia Civil, Museu do Trem, Museu dos Teatros do Rio de Janeiro, Museu Histórico e Diplomático Palácio Itamaraty, Museu Naval e Oceanográfico; em Niterói (RJ): Museu de História e Artes do Estado do Rio de Janeiro; em Petrópolis (RJ): Museu Imperial; e em Vassouras (RJ): Museu Casa da Hera.

${ }^{[4]}$ As fichas utilizadas como exemplo foram as do Museu D. João VI da Escola de Belas Artes/UFRJ e outras exemplificadas nos anais do Seminário Internacional Tecidos e sua Conservação no Brasil: museus e coleções, realizado no Museu Paulista - USP, em São Paulo (SP), em maio de 2006. Veja: Paula (2006).

${ }^{[5]}$ Respectivamente, Ida Catarina Lahmeyer, Laura Maria Lahmeyer e Cecilia Salomé Lahmeyer.

${ }^{[6]}$ No original: "(...) um effort constant pour faire parler les choses muettes, leur faire dire ce qu'elles ne disent pas d'elles mêmes, sur les hommes, sur les sociétés qui les ont produites, utilisées, transformées, façonnées à leur guise."

\section{REFERÊNCIAS}

BALFET, H. et al. Un essai de système descriptif du vêtement. In: DELAPORTE, Y. (Dir.). Vêtement et sociétés 2. Revue L'Ethnographie, Paris: Société d'Ethnographie, Tome LXXX, n. 92-93-94 p. 363-373, 1984.

\section{BARTHES, R. 0 sistema da moda. Lisboa: Ed. 70, 1981.}

BONADIO, M. C.; MATTOS, M. F. História e cultura de moda. São Paulo: Estação das Letras e Cores, 2011.

BOURDIEU, P. 0 poder simbólico. Lisboa: Difel, 1989.

BURGUELIN, O. Vestuário. In: Enciclopédia Einaudi, Soma/Psique - Corpo. Lisboa: Imprensa Nacional/Casa da Moeda, 1995, p. 337-362.

DUFLOS-PRIOT, M.-T. Une documentarion informatisée sur le costume traditionnel en France: le système descriptif du Musée National des Arts et Traditions Populaires. In: DELAPORTE, Y. (Dir.). Vêtement et sociétés 2. Revue L'Ethnographie, Paris: Société d'Ethnographie, Tome LXXX, n. 92-93-94, p. 375-381, 1984.

FEBVRE, L. Vers une autre histoire. Revue de Métaphysique et de Morale, 54e Année, n. 3-4, Les problèmes de l'histoire, Presses Universitaires de France, p. 225-247, jul./out. 1949. Disponível em: <http://www.jstor.org/stable/40899439>. Acesso em: 10 nov. 2013.

FERREIRA, A. B. H. Dicionário Aurélio da língua portuguesa. Curitiba: Positivo, 2010, p. 1152.

FERREZ, H. D.; BIANCHINI, M. H. S. Thesaurus para acervos museológicos. Rio de Janeiro: MinC/SPHAN/Pró-Memória, 1987. 2 v.

\section{GUIA DE MUSEUS BRASILEIROS. São Paulo: CPC-USP/Edusp/Imesp, 2000.}

MENESES, U. T. B. A cultura material no estudo das sociedades antigas. Revista de História, São Paulo: USP, n. 115, jul.-dez. 1983. Disponivel em: <http://revhistoria.usp.br/index.php/br/ edicoes/103-rh-115>. Acesso em: 10 nov. 2013.

PAULA, T. C. T. (Ed.) Tecidos e sua conservação no Brasil: museus e coleções. São Paulo: Museu Paulista da USP, 2006.

PROWN, J. D. Mind in matter: an introduction to material culture theory and method. Winterthur Portfolio, Chicago: The University of Chicago Press, v. 17, n. 1, p. 1-19, 1982. Disponivel em: <http://www.jstor.org/stable/1180761>. Acesso em: 10 nov. 2013.

ROCHE, D. História das coisas banais: nascimento do consumo XVII-XIX. Rio de Janeiro: Rocco, 2000. 